\title{
Accuracy Evaluation on the Respiration Rate Estimation using Off-the-shelf Pulse-Doppler Radar
}

\author{
Xingzhuo Li ${ }^{(l-2)}$, Shaoxuan $\mathrm{Li}^{(1-2)}$, Haobo $\mathrm{Li}^{(l)}$, Francesco Fioranelli( ${ }^{(I-2)}$ \\ ${ }^{(1)}$ School of Engineering, University of Glasgow, Glasgow, UK \\ ${ }^{(2)}$ Glasgow College UESTC, University of Electronic Science and Techonology of China, Chengdu, China \& University of Glasgow, \\ Glasgow, UK \\ francesco.fioranelli@glasgow.ac.uk
}

\begin{abstract}
This student paper presents preliminary results of using a pulse Doppler radar to detect the respiration rate of human subjects, examining the accuracy of the approach and evaluating the parameters to obtain the most precise result. In the study, the respiration data is recorded by repeatedly detecting people seated in front of the radar at different ranges as well as different aspect angles each time. Then Movement Target Indication, short-time Fourier transform and the analysis of the choice of doppler bins and window size of STFT are performed to evaluate the respiration rate and its precision. The results indicate that the respiration rate can be successfully detected at various ranges and angles and the relationship between Doppler bins and window size in processing is also observed to help us find the most accurate respiration rate.
\end{abstract}

Keywords-Pulse-Doppler radar, respiration detection, short-time Fourier transform, micro-Doppler

\section{INTRODUCTION}

Given the fast development of the miniaturization technology in electronics systems, as well as the next generation device networks like IOTs and 5G, Doppler radar based on the micro-Doppler effect has gained significant interest in detecting the micromotions of patients and elderly people [1]. In the context of hospital and in-home health monitoring, it is widely utilized in the daily motion tracking and classification, fall detection, sleep quality evaluation and baby care, and vital signs monitoring [2]. Besides these applications, Doppler radar has been reported as a novel contactless breathing rate monitoring technology to detect drivers' potential health problems [3]. Compared to other sensing technologies used in the respiration rate detection (e.g. wearable sensor, RGB sensor and depth camera), Doppler radar as a contactless, non-intrusive technology has the advantages of ignoring light conditions and no photo and video generation which may be perceived as privacy invasive.

In this student paper, we present some preliminary results of using an off-the-shelf pulse-Doppler radar system to detect the respiration rate of human subjects at different locations with respect to the radar in indoor environments. Simple algorithms based on spectrograms and peak-detection are demonstrated, highlighting the effect of different parameters on the final accuracy. Prior to applying the breathing rate detecting algorithms, the raw radar signal passes a band-stop filter to eliminate the clutter from static objects; this procedure is also known as Moving Target Indication (MTI). The radar we used is able to provide high range resolution (a few $\mathrm{cm}$ ) to characterise information involving chest movement due to respiration. Additionally, it is possible to use the relevant Doppler signature to estimate the breathing rate and characterize the breathing strength.

This paper is organized as follows: section II describes the radar used in this work and experimental setup. Section
III explains the radar signal processing procedures (MTIfiltering and STFT), and then discusses the preliminary results about the breathing rate estimation with participants at different positions. Section IV summarizes the theoretical and technical work, and besides that, some future applications are indicated.

\section{DATA COLlECTION AND EXPERIMENT DESIGN}

In the experiment, a pulse-Doppler radar is used in the recording for its capabilities of detecting both range and Doppler information on the subjects. The radar processing applies pulse compression to get higher detection ability in range [5], and Doppler effect enables to observe the shifted frequency caused by the signal reflection of the moving target, in this case the movement of the chest and abdomen of the subjects while breathing. The radar used in this study was the commercial off-the-shelf Ultra-Wide Band (UWB) model Xethru by Novelda. The Pulse Repetition Frequency used was $17 \mathrm{~Hz}$ as the respiration rate is a slow movement, not requiring a high PRF for unambiguous Doppler measurements.

The data were recorded according to the sketch measurement plan in Figure 1, based on an MSc student project at the University of Glasgow [6]. The radar recorded the breathing information of two subjects, individually sitting at different distances away from radar to obtain data from several ranges for accuracy evaluation, namely from $1 \mathrm{~m}$ to $5 \mathrm{~m}$ with a recording every $1 \mathrm{~m}$. Afterwards recordings were repeated to obtain respiration data at two different aspect angles to the radar (approximately 18 degrees and 36 degrees) [6]. By using the pulse Doppler radar, the recorded data contains both range information which can indicate where the motions are captured in range analysis, and Doppler information which indicates the recorded respiration times and rates. The duration of each recording was $35 \mathrm{~s}$ and the subjects were performing three different styles of breathing, namely slow respiration, normal, and fast breathing.

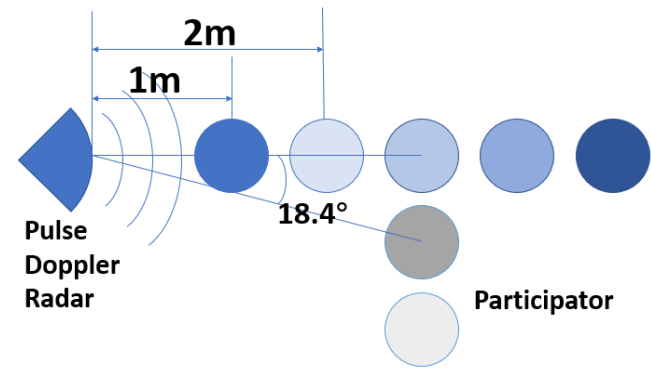

Fig. 1. Sketch of the experimental setup for respiration rate estimation, with positions of the subject indicated at different ranges and aspect angles

\section{DATA PROCESSING AND RESUlT}

After obtaining the raw radar data (sequences of received 
radar pulses), they are processed following the steps represented in Figure 2. First a Fast Fourier Transform is applied with MTI filter to identify the range bins containing the subject's signature by looking at the range-Doppler maps. Subsequently, Short Time Fourier Transform (STFT) is applied on the selected range bins with a suitable choice of parameters (window length and overlap) to obtain spectrogram plots, i.e. Doppler vs time patterns. Finally, a number of Doppler bins in the spectrogram plots are selected to estimate the oscillations and peaks of the signal levels due to the respiration movements.

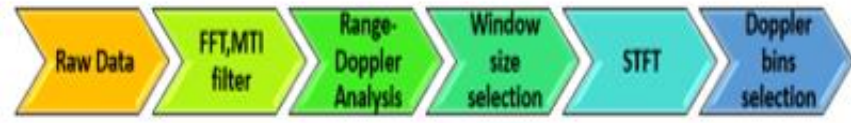

Fig. 2. Block diagram showing the radar signal processing steps of this work

\section{A. Preprocessing}

In the processing step, the radar data are first combined and reorganized, then the Fast Fourier Transform (FFT) and MTI filter are applied to extract Doppler information while suppressing clutter noise from stationary objects. Then the range-Doppler diagram can indicate the distance from the radar of the breathing detected and locate the range bins needed for Short-Time Fourier Transform with proper window size to plot Doppler-Time figures, spectrograms. In Doppler-Time analysis, specific Doppler bins containing respiration rate can be chosen to estimate the detected respiration rate.

\section{B. Short-Time Fourier Transform Process}

Short-time Fourier transform works by applying an FFT across short, overlapped window on the received radar signal in the time domain, in order to perform timefrequency analysis [7]. This was implemented in MATLAB in this work, with the view of developing lighter implementation in terms of computational requirements in Python and $\mathrm{C}++$ at a later stage. The most important parameter for the STFT is the duration of the window of each FFT, which directly influences the time resolution of the final result and the Doppler resolution. There is trade-off between them, whereby shorter windows will provide finer time resolution but coarser Doppler resolution, and the other way round. Two examples of spectrograms resulting from STFT are shown in Figure 3, using two different durations of the window, 40 and 25 pulses respectively. The three different styles of breathing are visible in the figures, with increasing Doppler spreading as the respiration velocity increases, i.e. the velocity of the chest movement.

\section{Micro-Doppler and Doppler Bins Process}

The STFT processing enables to detect and characterize the small frequency shifts caused by the small amplitude movements of the subjects' chest during the respiration. This can be considered an example of micro-Doppler signal [8] C. Li has discussed that the expected respiration rate for an adult human in normal health conditions is between 0.1 and $1 \mathrm{~Hz}$ in terms of its frequency, and this can be measured by counting peaks in the relevant RF signal [9]. After STFT processing, the information on the micro-Doppler signature at 1 Doppler $\mathrm{Hz}$ could symbolize the displacement of chest and the breath of human could be detected, as shown in the example in Figure 4a. Therefore, the reflection of the motion in the chest is the periodic fluctuation of the waveform of the Doppler Frequency Response-Time graph and the number of breaths can be detected by counting the number of peaks.
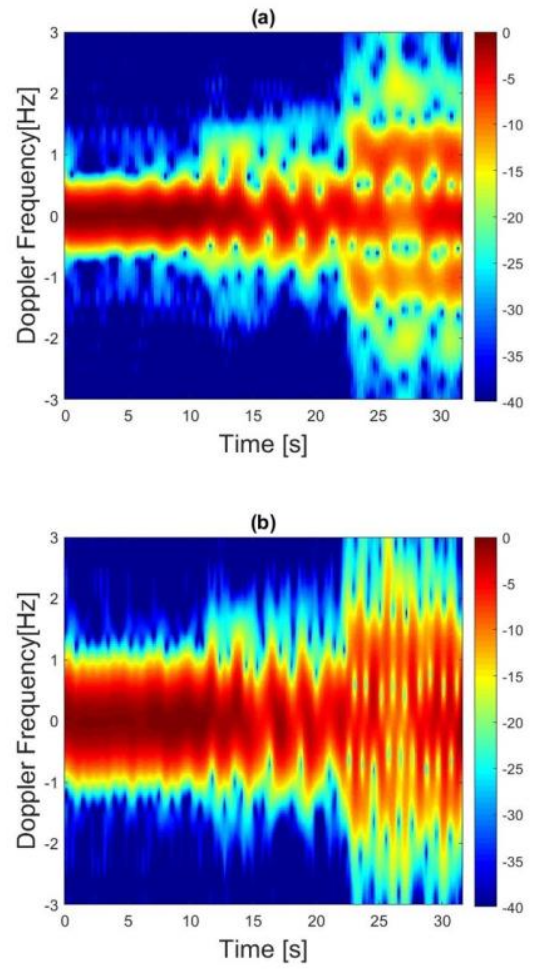

Fig. 3. Example of spectrograms: (a) Doppler-Time spectrograms of respiration information when window size is 40 pulses; (b) Doppler-Time spectrograms of respiration information when window size is 25 pulses.
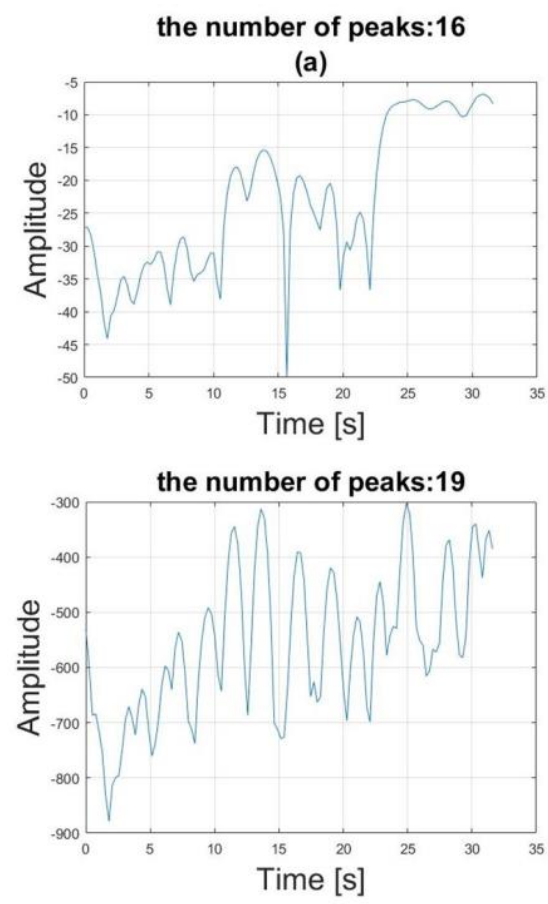

Fig. 4. Doppler frequency response of chest at $1 \mathrm{~Hz}$ (a), accumulation of frequency response of chest from 0 to $1 \mathrm{~Hz}$ (b)

However, in this figure, noise takes the leading effect and the regular, oscillatory behavior expected for respiration cannot be easily seen. To solve this, Doppler bins from zero 
to target bin were accumulated to enhance the desired peaks so that the effect of noise could be reduced, and the accumulated result is showed in figure $4 b$.

The respiration rate detected at different distances using a default STFT window size of 40 radar pulses and two values of accumulated Doppler bins is shown in Table 1. The precision and noise variation caused by the choice of window size and Doppler bins lead to the deviation of the detected respiration rate from the real rate noted during the data collection. In this way, following analysis is performed to find out the relationship between these two parameters and the most suitable parameters for accurate detection.

TABLE I. RESPIRATORY RATE USING DEFAULT STFT WINDOW SIZE (40 PULSES) AND DOPPLER BINS (ACCUMULATION UP TO 0.9 OR 1 HZ)

\begin{tabular}{|c|c|c|c|c|c|c|c|c|c|c|}
\hline \multicolumn{11}{|c|}{ Respiratory Rate in Different Window Size and Doppler Bins } \\
\hline \multicolumn{2}{|c|}{ Range } & 1 & 2 & 3 & 4 & 5 & shift $1^{*}$ & shift $2^{*}$ & Meter & Average \\
\hline \multicolumn{2}{|c|}{ Actual Respiration Rate } & 26 & 22 & 24 & 25 & 24 & 24 & 23 & Times/30s & Deviation \\
\hline \multicolumn{11}{|c|}{ window size Doppler Bins } \\
\hline \multirow{2}{*}{40} & 0.9 & 26 & 19 & 20 & 21 & 20 & 21 & 20 & Times/30s & $12.64 \%$ \\
\hline & 1 & 26 & 21 & 20 & 19 & 20 & 20 & 20 & Times/30s & $13.08 \%$ \\
\hline
\end{tabular}

\section{Window Size and Dopple Bins Selection}

As fig. 4 (b) shows, some glitches in peak identification can still disturb the result. As discussed earlier, the window size is a significant factor in STFT processing. On the other hand, the extent of the accumulated Doppler bins is also an essential factor to take into account. Therefore, experiments on MATLAB processing have been done to evaluate the effect of these parameters. Figure 5 shows the number of peaks detected from the spectrogram for different STFT window size and accumulating different Doppler Frequency bins. Figure 5a shows the number of detected peaks as a function of these parameters, whereas figure $5 \mathrm{~b}$ shows with a binary plot the pairs of parameters values providing the correct result, i.e. the estimated respiration rate equal to the ground truth noted during the data collection.

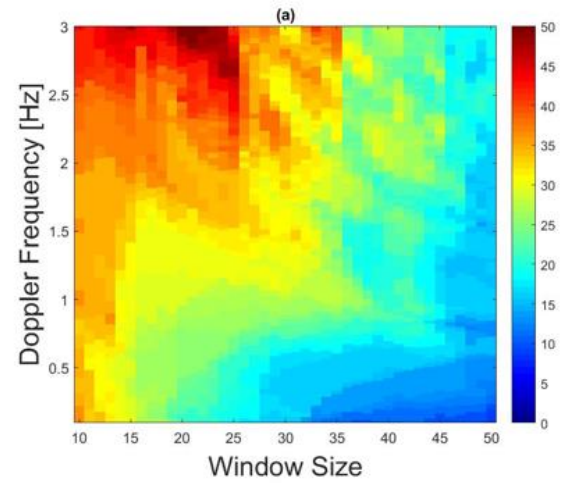

(b)

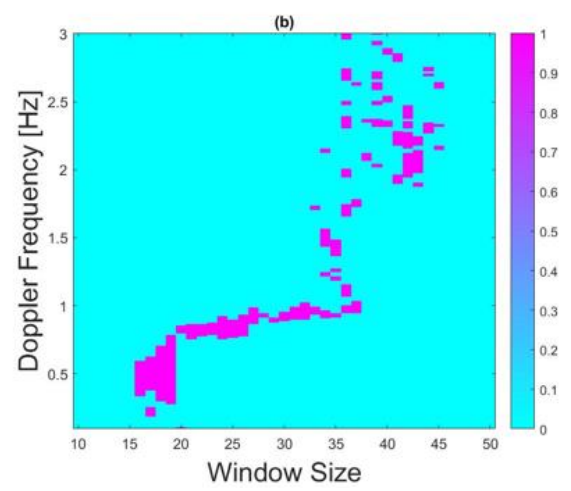

Fig. 5. Heatmap of the number of detected respiration peaks for different STFT window size and Doppler bins (a), and correct values highlighted in purple for the different parameter combinations (b)

To be more accurate, multiple data files ( 5 in this case) were used to perform the same test and the results are shown in figure 6a. The value plotted is the number of correct estimations of the respiration rate, from 0 to 5 , for different combinations of STFT window duration and accumulated Doppler bins. From this figure, the most accurate area is around 0.9 Doppler $\mathrm{Hz}$ and 25-35 pulses window size. Figure $6 \mathrm{~b}$ shows the number of correct detections accepting a $5 \%$ error, i.e. 1 possible wrong estimation out of 5 . In this figure, the correct detections for the five tests are still in the range of 0.85 to 0.94 (Doppler frequency) and 25 to 35 (STFT window size).

To verify the feasibility and accuracy of the parameters found to be the most accurate ones from figure $6 \mathrm{~b}$, the process is carried out again to find respiration rate when the window size is 28 and doppler bins are 0.9 or $0.92 \mathrm{~Hz}$, and their deviations. The results are shown in Table 2 and a result with greater accuracy and fewer deviation can be obtained.

\section{CONCULUSION}

In this student paper, the analysis is performed to estimate the respiration rate using pulse Doppler radar and evaluate its variation at different ranges of the subjects from the radar, as well as identify the most useful or accurate STFT windows size and Doppler bins accumulated in the spectrogram.

Future work includes using multiple Xethru radars, recruiting more volunteers for data collection and applying a classifier (e.g. Support Vector Machine and neural network) to characterize different levels of breathing. For the hardware part, building a fast, reliable breathing detection system containing sensing (radar and other possible sensors) and signal processing (e.g. FPGA, Raspberry Pi) units is the next step and this will significantly improve the precision of remote health evaluation.

\section{ACKNOWLEDGMENT}

The authors would like to thank Jiaqian Peng for his support in the data collection. The acquisition of the Xethru radar system and its validation activities were supported by the SFC through Interface voucher and the UK EPSRC through grant EP/R041679/1, INSHEP. 

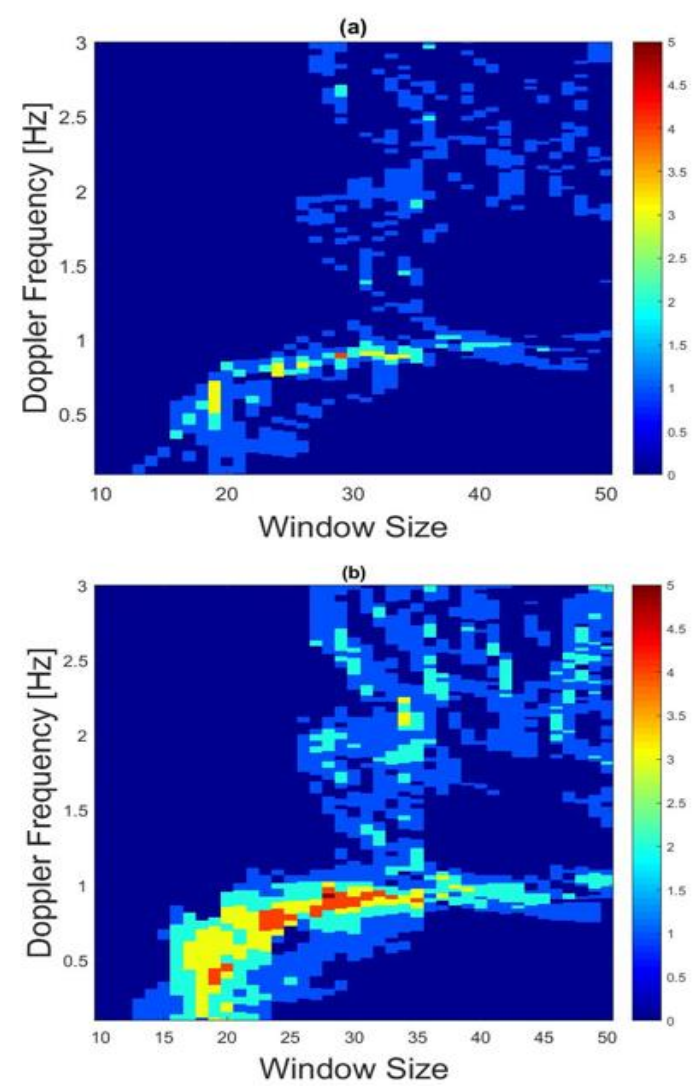

Fig. 6. The number of correct respiration detections for different window size and Doppler bins (a) and the same number with 5\% error tolerance to right rates (b).

\section{REFERENCES}

[1] O. Postolache, P. S. Girão, R. N. Madeira and G. Postolache, "Microwave FMCW Doppler radar implementation for in-house pervasive health care system," 2010 IEEE International Workshop on Medical Measurements and Applications, Ottawa, ON, 2010, pp. 4752.

[2] G. Sacco, E. Pittella, E. Piuzzi and S. Pisa, "A radar system for indoor human localization and breath monitoring," 2018 IEEE International Symposium on Medical Measurements and Applications (MeMeA), Rome, 2018, pp. 1-6.

[3] Yang, Zhicheng \& Bocca, Maurizio \& Jain, Vivek \& Mohapatra, Prasant. (2018). Contactless Breathing Rate Monitoring in Vehicle Using UWB Radar. 13-18.

[4] W. Yuegang, J. Shao and X. Hongtao, "Non-stationary Signals Processing Based on STFT," 2007 8th International Conference on Electronic Measurement and Instruments, Xi'an, 2007, pp. 3-301-3304

[5] Z. Wang, H. Han, M. Liu and Q. Yang, "Modeling and simulation of pulse Doppler radar system based on SystemVue code," 2017 International Applied Computational Electromagnetics Society Symposium (ACES), Suzhou, 2017, pp. 1-2.

[6] J. Peng, "Assessment Capabilities of Short-ranged Commercial Radar Sensors", MSc degree thesis, School of Engineering, University of Glasgow, unpublished.

[7] Y. Zhao, Z. Zou, L. Wu and Y. Li, "Frequency Detection Algorithm for Frequency Diversity Signal Based on STFT," 2015 Fifth International Conference on Instrumentation and Measurement, Computer, Communication and Control (IMCCC), Qinhuangdao, 2015, pp. 790-793.

[8] Y. C. Yang, N. N. Tong, C. Q. Feng and S. S. He, "Micro-Doppler extraction of rotating targets based on Doppler rate," 2011 IEEE International Conference on Signal Processing, Communications and Computing (ICSPCC), Xi'an, 2011, pp. 1-4.

[9] C. Li and J. Lin, "Optimal Carrier Frequency of Non-contact Vital Sign Detectors," 2007 IEEE Radio and Wireless Symposium, Long Beach, CA, 2007, pp. 281-284.

TABLE II. RESPIRATORY RATE FOR MORE ACCURATE WINDOW SIZE (28 PULSES) AND DOPPLER BINS ( 0.92HZ AND 0.9HZ) CHOSEN FORM THE ANALYSIS

\begin{tabular}{|c|c|c|c|c|c|c|c|c|c|c|}
\hline \multicolumn{11}{|c|}{ Respiratory Rate in Different Window Size and Doppler Bins } \\
\hline \multicolumn{2}{|c|}{ Range } & 1 & 2 & 3 & 4 & 5 & shift $1^{*}$ & shift $2^{*}$ & Meter & Average \\
\hline \multicolumn{2}{|c|}{ Actual Respiration Rate } & 26 & 22 & 24 & 25 & 24 & 24 & 23 & Times/30s & Deviation \\
\hline \multicolumn{11}{|c|}{ window size Doppler Bins } \\
\hline \multirow{2}{*}{28} & 0.9 & 26 & 22 & 24 & 25 & 23 & 24 & 22 & Times/30s & $1.21 \%$ \\
\hline & 0.92 & 26 & 21 & 23 & 24 & 23 & 26 & 22 & Times/30s & $4.22 \%$ \\
\hline
\end{tabular}

